\title{
Malignancies in Italian Patients with Systemic Sclerosis Positive for Anti-RNA Polymerase III Antibodies
}

\author{
PAOLO AIRO', ANGELA CERIBELLI, ILARIA CAVAZZANA, MARA TARABORELLI, STEFANIA ZINGARELLI, \\ and FRANCO FRANCESCHINI
}

ABSTRACT. Objective. To evaluate the frequency of malignancies in Italian patients with systemic sclerosis (SSc) and anti-RNA polymerase III (RNAP III), antitopoisomerase I (topo I), or anticentromere antibodies (ACA); and to characterize the temporal relationship between the 2 diseases, in order to confirm data suggesting a close temporal relationship between the onset of SSc and malignancy in American patients with anti-RNAP III antibodies.

Methods. From a cohort of 466 consecutive SSc patients, 360 Italians with isolated positivity for anti-RNAP III $(\mathrm{n}=16)$, anti-topo I $(\mathrm{n}=101)$, or ACA $(\mathrm{n}=243)$ were identified. Malignancy cases were divided according to their relationship with SSc onset into 3 categories: preceding, synchronous with, or metachronous to the onset of SSc (diagnosed more than 6 months before; 6 months before to 12 months after; and more than 12 months after onset of SSc, respectively).

Results. Malignancies were more frequent in the anti-RNAP III group (7/16 patients), than in the anti-topo I (11/101) and ACA groups $(21 / 243)(\mathrm{p}<0.001)$. This difference was accounted for by the number of patients with cancer synchronous to the onset of SSc (3/16 in the anti-RNAP III group vs $0 / 101$ in the anti-topo I and $1 / 243$ in the ACA group; $p<0.001$ ), whereas neither the number of malignancies preceding nor those metachronous to the onset of SSc was significantly different between the groups.

Conclusion. In a cohort of Italian patients with SSc we observed a significant association between malignancies synchronous to SSc onset and positivity for anti-RNAP III antibodies, similar to that described in American patients with SSc. (First Release April 1 2011; J Rheumatol 2011; 38:1329-34; doi:10.3899/jrheum.101144)

Key Indexing Terms:

SYSTEMIC SCLEROSIS MALIGNANCY ANTITOPOISOMERASE I ANTIBODIES

In systemic sclerosis (SSc), autoantibodies are seen in more than $95 \%$ of patients. At least 7 SSc-related autoantibodies that are markers of clinically and genetically distinct subsets have been described ${ }^{1}$. Although they are not known to have a distinct role in the pathogenesis of the disease, their possible etiologic role has been suggested ${ }^{1}$.

Anti-RNA polymerase III (RNAP III) antibodies are associated with diffuse cutaneous involvement $(\mathrm{dcSSc})$ and with other clinical features of SSc such as renal $\mathrm{crisis}^{2,3}$ and gastric antral vascular ectasia (GAVE) ${ }^{4,5}$. Interestingly, in patients with anti-RNAP III antibodies the onset of the disease is often very rapid, and the progression of skin involvement appears to be faster than in other patients with dcSSc,

From the Rheumatology and Clinical Immunology Service, Spedali Civili and University of Brescia, Brescia, Italy.

P. Airo', MD, Rheumatology and Clinical Immunology Service, Spedali Civili, University of Brescia; A. Ceribelli, MD, Department of Oral Biology, University of Florida, Gainesville, Florida, USA; I. Cavazzana, $M D ; M$. Taraborelli, MD; S. Zingarelli, MD; F. Franceschini, MD, Rheumatology and Clinical Immunology Service, Spedali Civili, University of Brescia.

Address correspondence to Dr. P. Airo', Rheumatology and Clinical Immunology Service, Spedali Civili di Brescia, piazzale Spedali Civili 1, 25123 Brescia, Italy.E-mail: airo@bresciareumatologia.it

Accepted for publication February 4, 2011.

\section{ANTI-RNA POLYMERASE III ANTIBODIES ANTICENTROMERE ANTIBODIES}

such as those with anti-topoisomerase I (topo I) antibodies $^{1,6}$. Accordingly, renal crisis usually develops earlier during the disease course in anti-RNAP III-positive patients compared to the anti-topo I subset ${ }^{7}$. Finally, the onset of GAVE in patients with anti-RNAP III is usually in the first year of disease ${ }^{5}$.

A close temporal relationship between the onset of SSc and that of malignancy has recently been described in some patients in the USA with anti-RNAP III antibodies ${ }^{8}$. This relationship was significantly different from one observed in patients with other autoantibody specificities ${ }^{8}$. Since RNAP III antigenic expression by tumoral tissues was also demonstrated, these observations led to the important suggestion that malignancy may initiate a specific immune response against RNAP III, leading to a rapid onset and progression of SSc in some patients ${ }^{8}$. It is known that autoantibody distributions in cohorts of SSc patients from the US and continental Europe are significantly different ${ }^{9}$, and in particular, anti-RNAP III antibodies and their complications such as renal crisis are relatively rare in France and Italy $7,9,10,11$. For this reason, the relationship between cancer and the subset of anti-RNAP III-positive patients with SSc observed in the US needs to be confirmed in other populations.

Personal non-commercial use only. The Journal of Rheumatology Copyright @ 2011 . All rights reserved. 
We evaluated the frequency of malignancies in our series of Italian patients with SSc and the temporal relationship between the 2 diseases, and we compared the groups with isolated positivity of one of the 3 more frequent SSc-specific autoantibodies: anti-RNAP III, anti-topo I, and anticentromere (ACA).

\section{MATERIALS AND METHODS}

Patients. Patients were selected among a series of 466 consecutive patients with SSc followed at our unit as inpatients or as outpatients from 1988 to August 2010, with at least 2 visits. Patients provided written informed consent to their data treatment.

Patients were classified as having SSc according to the 2001 criteria proposed by LeRoy and Medsger ${ }^{12}$ that include patients with early SSc, identified by nailfold capillaroscopy and by specific autoantibody tests. Only patients with limited SSc (ISSc) according to LeRoy and Medsger (i.e., patients without definite sclerodactyly or other cutaneous changes) were excluded from this study. The definition of limited cutaneous SSc (lcSSc) according to LeRoy and Medsger includes also a proportion of patients not satisfying the 1980 American College of Rheumatology (ACR) criteria $^{13}$ : these are patients with sclerodactyly plus specific nailfold capillaroscopic abnormalities and/or autoantibodies, but without other minor ACR criteria (digital pitting scars/loss of substance, or bibasilar pulmonary fibrosis). Patients with sclerodermatous changes proximal to the elbows and knees at any time in their clinical history were defined as having diffuse cutaneous SSc $(\mathrm{dcSSc})^{12}$. Indeed, a proportion of patients did not meet ACR criteria (10\% of the anti-topo I and $39 \%$ of the ACA group, but none of the anti-RNAP III group).

Disease onset was defined by the onset of the first symptom other than Raynaud's phenomenon. At our institution patients with SSc are routinely evaluated according to standard procedures, analogous to those recommended by a consensus conference for the identification of a core set of variables for SSc studies ${ }^{14}$. Interstitial lung disease was defined by chest radiograph or high resolution computer tomography. The latter was performed in patients with unexplained dyspnea or abnormal pulmonary function tests. Before 2003, the diagnosis of pulmonary hypertension was made in patients with very high (usually $>60 \mathrm{~mm} \mathrm{Hg}$ ) and unexplained (no significant ILD; no pulmonary thromboembolism) systolic pressure on Doppler echocardiography. Afterwards, pulmonary arterial hypertension was diagnosed by right-heart catheterization (RHC), for values $>25 \mathrm{~mm}$ $\mathrm{Hg}$ at rest. RHC was performed in patients with high systolic pressure on Doppler echocardiography (> $45 \mathrm{~mm} \mathrm{Hg}$ ) screening test, or with unexplained dyspnea. Disease severity was evaluated by Medsger severity scales $^{15}$

Serologic analysis. The serum of all SSc patients was analyzed by indirect immunofluorescence test using commercial HEp-2 slides (BioRad, Hercules, CA, USA; Inova Diagnostics, San Diego, CA, USA) to evaluate $\mathrm{ACA}$, considered positive at titer $\geq 1: 160$, and by counterimmunoelectrophoresis, using rabbit thymus and human or porcine spleen extracts as substrates to evaluate anti-topo I antibodies. Anti-RNAP III antibodies were evaluated by commercial ELISA kits, with recombinant antigen (MBL, Nagoya, Japan; Inova) in 262 of the 466 patients. Notably, anti-RNAP III antibodies were tested in 85 out of 110 sera negative for anti-topo I and ACA, but further sera samples were not available in 25 of these patients. Autoantibody determination was performed at least twice in almost all patients ( $>90 \%$ in the anti-topo I and the ACA group; $56 \%$ in the anti-RNAP III group). We did not detect any shift from one of 3 SSc-specific antibodies to another during the illness. This change is generally considered exceptional (as reviewed ${ }^{1,16}$ ). The serum sample closest to the diagnosis of cancer was considered in this study, or the last sample for patients without malignancy. The median duration between cancer diagnosis and investigation of the serum sample in question was 13 months.
Chart review for malignancies. We excluded the following from the study: patients of ancestry other than Italian; patients with overlap syndromes with polymyositis; and patients in whom more than one of the 3 autoantibody specificities was present in combination.

The clinical charts of 360 Italian patients with isolated positive tests for anti-RNAP III, anti-topo I, or ACA were included in the study and reevaluated to identify those with a diagnosis of malignancy. The date of cancer diagnosis was defined by the pathology report (or hospital discharge letters) in all cases, except for 2 in which malignancy diagnosis preceded onset of SSc by a long period, and was reported by the general practitioner with only the year indicated. In these latter cases, clinical or laboratory data confirmed the diagnosis.

Malignancy cases were divided according to their relationship with onset of SSc into 3 categories: (1) malignancies diagnosed more than 6 months before the onset of SSc ("cancer preceding the onset of SSc"); (2) malignancies diagnosed 6 months before to 12 months after onset of SSc ("cancer synchronous to the onset of SSc"); and (3) malignancies diagnosed more than 12 months after the onset of SSc ("cancer metachronous to the onset of SSc"). The time intervals for the definition of cancer synchronous to the onset of SSc were chosen considering possible delays in the identification of cancer that might have been present before the onset of SSc and diagnosed afterwards.

Statistical analysis. If not otherwise indicated, data are presented as the median (10th-90th percentile). Clinical and demographic characteristics were compared by means of chi-square or Fisher's exact for categorical data, and by Kruskal-Wallis test for continuous variables.

\section{RESULTS}

Among 466 consecutive patients with SSc followed at our institution, $460(98.7 \%)$ were of Italian ancestry. Isolated anti-RNAP III antibodies, anti-topo I antibodies, and ACA were found in 17, 104, and 243 of them, respectively, whereas 3 sera were positive for combinations of antibodies (2 with anti-RNAP III and anti-topo I antibodies; one with anti-RNAP III and ACA) and 93 were negative for all 3 specificities. These latter 2 groups of patients were excluded from further analysis to avoid the confounding effect of double specificities, and because not all sera negative for anti-topo I antibodies and ACA were tested for anti-RNAP III antibodies (see Materials and Methods). Moreover, one patient with anti-RNAP III antibodies and one with anti-topo I antibodies were excluded because the clinical diagnosis overlapped with polymyositis, and cancer risk might be influenced by the diagnosis of myositis and not by the SSc-related antibody. Two patients (both with anti-topo I antibodies) were excluded because of insufficient clinical data in the chart review.

Thus, 360 Italian patients with isolated anti-RNAP III, anti-topo I, or ACA were studied (Figure 1). Their demographic and clinical data are presented in Table 1. Median time of followup of the group evaluated was 118 months, for a total of 4041 patient-years. The followup of patients with anti-RNAP III antibodies was shorter than that for other groups $(p=0.037)$, possibly reflecting the more recent availability of the diagnostic test, but there were no other significant differences between the anti-RNAP III and anti-topo I groups. As expected, patients with ACA were more frequently female, were less frequently affected with 


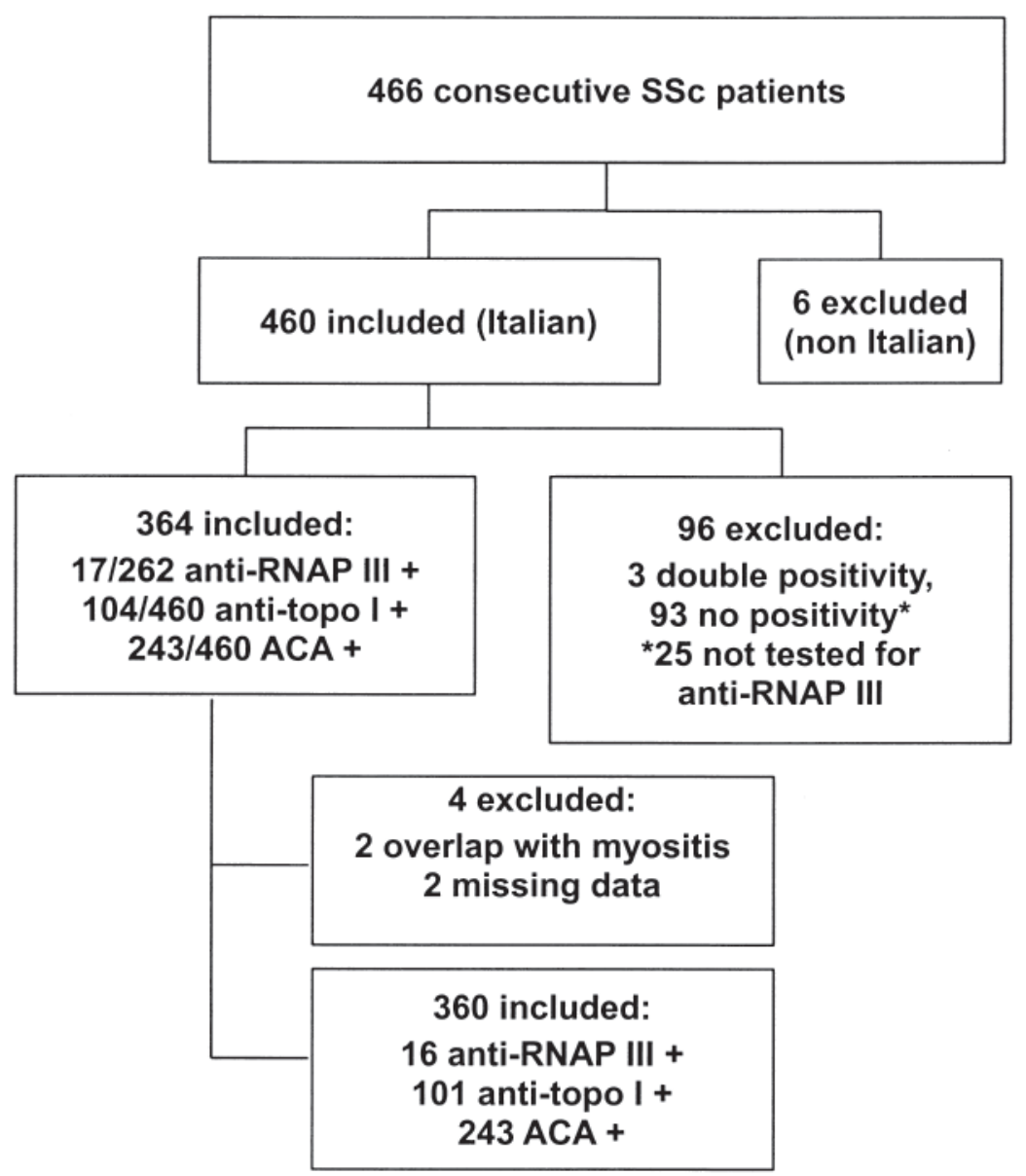

Figure 1. The selection process: 360 Italian patients with isolated anti-RNAP III, anti-topo I, or ACA were selected for study.

Table 1. Main demographic and clinical features of 360 Italian patients with SSc in the study. Data are presented as median (10th-90th percentile) or number $(\%)$.

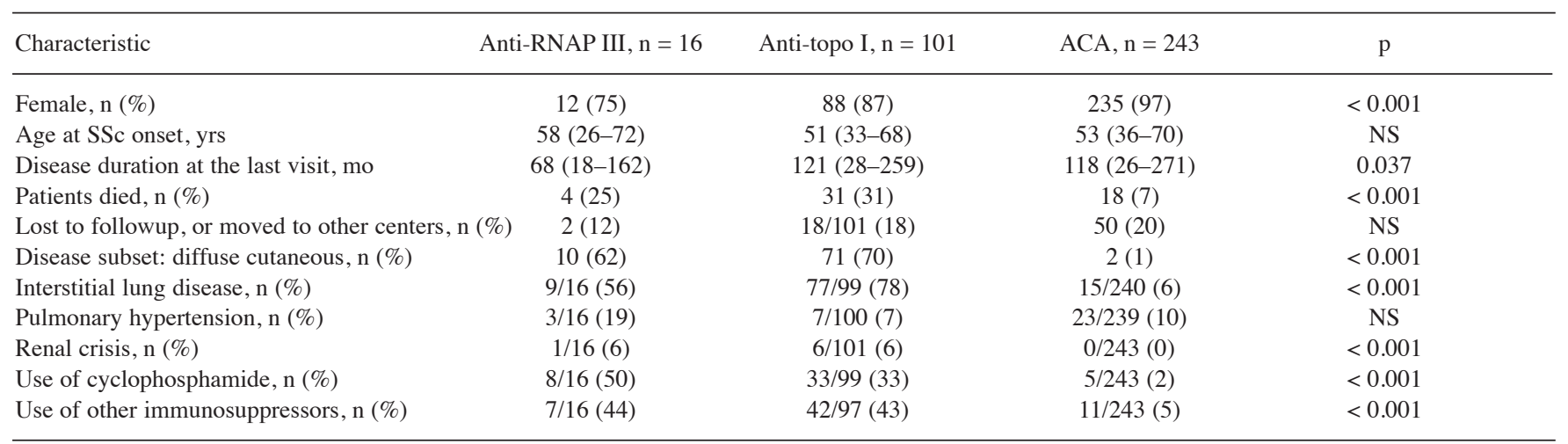

NS: nonsignificant

dcSSc, interstitial lung involvement and renal crisis, and were less frequently treated with cyclophosphamide or other immunosuppressive drugs.

As shown in Table 2, a history of malignancy was present in $39(10.8 \%)$ out of $360 \mathrm{SSc}$ patients included in the study. One patient in the ACA group had 2 different malignancies: non-Hodgkin lymphoma diagnosed before SSc, and breast cancer diagnosed after SSc.

The frequency of malignancy was significantly higher in the anti-RNAP III group [7 out of 16 (95\% confidence interPersonal non-commercial use only. The Journal of Rheumatology Copyright @ 2011 . All rights reserved. 
Table 2. Neoplastic history in 360 Italian patients with SSc in the study. Data are numbers (\%).

\begin{tabular}{lcccc}
\hline Characteristic & $\begin{array}{c}\text { Anti-RNAP III, } \\
\mathrm{n}=16\end{array}$ & $\begin{array}{c}\text { Anti-topo I, } \\
\mathrm{n}=101\end{array}$ & $\begin{array}{c}\text { ACA, } \\
\mathrm{n}=243\end{array}$ & $\mathrm{p}$ \\
\hline Malignancy total number & $7(43.8)$ & $11(10.9)$ & $21(8.6)$ & $<0.001$ \\
Cancer preceding onset of SSc & $1(6.2)$ & $2(2.0)$ & $9(3.7)^{*}$ & $\begin{array}{c}\mathrm{NS} \\
\text { Cancer synchronous to onset of SSc }\end{array}$ \\
Cancer metachronous to onset of SSc & $3(18.8)$ & $0(0)$ & $1(0.4)$ & $<0.001$ \\
\hline
\end{tabular}

* One patient in the ACA group had 2 malignancies. NS: nonsignificant.

val $23.1 \%-66.9 \%)]$ than in the anti-topo I $(11 / 101,10.9 \%)$ and the ACA groups $(21 / 243,8.6 \%$; $p<0.001)$.

The difference between the groups was accounted for by the number of anti-RNAP III patients in which diagnosis of cancer was synchronous (6 months before to 12 months after) with the onset of SSc: $3 / 16$ compared to $0 / 101$ in the anti-topo I group and 1/243 in the ACA group ( $p<0.001)$; whereas the number of patients in which a cancer diagnosis preceded the onset of SSc, or was made more than 1 year after the onset of SSc, did not differ significantly between the groups (Table 2). The time intervals for the definition of cancer synchronous to the onset of SSc were chosen considering possible delays in the identification of cancer that might have been present before the onset of SSc and diagnosed afterwards. As a further precaution, an analysis was also performed including malignancies diagnosed between 12 and 36 months after the onset of SSc in the definition of cancer synchronous to the onset of SSc; but the results did not change (data not shown; $\mathrm{p}<0.001$ ).

The characteristics of the 4 patients with cancer synchronous to SSc are presented in Table 3. Notably, in Patient 3, the histotype of the esophageal cancer was squamous cell carcinoma of the pharyngeal esophagus. This cannot be explained by chronic gastric reflux, which can cause Barrett's esophagus and adenocarcinoma of the lower esophagus in patients with long-standing SSc. In all 3 patients with anti-RNAP III antibodies the onset of SSc was very rapid, with no interval between the appearance of Raynaud's phenomenon and a fast progression to diffuse skin involvement [Rodnan Skin Score (RSS) range: 14-51], that did not regress in any of them during the followup (range 22-35 months since onset of SSc). Moreover, severe organ involvement was found in all of them (Table 3). Among these 3 patients, the lowest RSS was observed in one patient treated with rituximab for non-Hodgkin lymphoma, a therapy that may also reduce the skin score of patients with $\mathrm{SSc}^{17}$. Since 2 of the cancer patients died soon after onset of SSc, the natural history of their skin over time cannot be determined, and it is therefore difficult to draw any definitive conclusions on the issue of SSc disease severity in these patients. In the other 13 anti-RNAP III-positive patients, the peak RSS (median 21, range 4-40) was observed at a median of 18 months (range 1-76) after onset of SSc, and then decreased in most of them (at the last observation, median 5.5 months, range 3-13).

The diagnosis of malignancy preceded the onset of SSc in one patient with anti-RNAP III antibodies (breast cancer diagnosed 54 months before onset of SSc), in 2 patients with anti-topo I antibodies (chronic lymphocytic leukemia and thyroid follicular adenocarcinoma, diagnosed 236 and 72 months, respectively, before SSc onset), and in 9 patients with ACA (4 with breast cancer; 1 each with non-Hodgkin lymphoma, essential thrombocytemia, uterine cervix, and

Table 3. Clinical characteristics of patients with diagnosis of malignancy synchronous to the onset of SSc.

\begin{tabular}{|c|c|c|c|c|c|c|c|c|}
\hline Patient & Antibody & Sex & $\begin{array}{l}\text { Age at } \\
\text { SSc Onset, } \\
\text { yrs }\end{array}$ & $\begin{array}{c}\text { SSc Duration at } \\
\text { Cancer Diagnosis, } \\
\text { mo }\end{array}$ & Cancer Type & $\begin{array}{c}\text { Followup After Cancer } \\
\text { Diagnosis (mo) } \\
\text { Outcome }\end{array}$ & $\begin{array}{l}\text { Maximum } \\
\text { RSS }\end{array}$ & $\begin{array}{c}\text { Severe SSc } \\
\text { Organ Involvement* }\end{array}$ \\
\hline 2 & RNA pol III & $\mathrm{F}$ & 72 & -3 & $\begin{array}{c}\text { Ductal carcinoma, } \\
\text { breast }\end{array}$ & $\begin{array}{l}26 \text { (alive, receiving hormone } \\
\text { therapy) }\end{array}$ & 51 & Skin, kidney, GAVE \\
\hline 4 & ACA & F & 44 & -2 & $\begin{array}{c}\text { Ductal carcinoma, } \\
\text { breast }\end{array}$ & $\begin{array}{l}18 \text { (alive, after chemo- and } \\
\text { radiotherapy; receiving } \\
\text { hormone therapy) }\end{array}$ & 4 & Vascular \\
\hline
\end{tabular}

\footnotetext{
* Medsger severity scales $\geq 3^{15}$ or gastric antral vascular ectasia (GAVE). RSS: Rodnan Skin Score.
} Personal non-commercial use only. The Journal of Rheumatology Copyright @ 2011 . All rights reserved. 
thyroid and bladder cancer; range 78-206 months before SSc onset).

More often, diagnosis of malignancy was made in patients with a long history of SSc: in 3 cases in the antiRNAP III group (1 each with prostate, breast, and biliary duct carcinoma; interval between SSc onset and cancer diagnosis, range 25-171 months), in 9 cases in the anti-topo I group (lung cancer, 3; colon, 2; stomach, breast, bladder, uterine cervix, 1 each; interval between SSc onset and cancer diagnosis, range 25-266 months), and 12 cases in the ACA group (breast cancer, 6; non-Hodgkin lymphoma, colon, lung, ovary, vulvar, and hepatocellular carcinoma, 1 each; interval between SSc onset and cancer diagnosis, range 18-316 months).

Exposure to cyclophosphamide might be relevant for cancers metachronous to SSc. None of the 3 patients with anti-RNAP III antibodies and metachronous cancer were exposed to cyclophosphamide, as compared with $3 / 9$ in the anti-topo I group and 0/12 in the ACA group (p: not significant).

\section{DISCUSSION}

An increased risk of cancer among patients with SSc has been demonstrated by nationwide population-based studies from Northern Europe ${ }^{18,19}$. Possible explanations for the association of the 2 diseases are: common risk factors, such as exposition to occupational risk factors like silica dust or solvents; exposure to immunosuppressive drugs; effects of chronic inflammation and fibrosis; possible genetic predispositions to both diseases ${ }^{18,19}$. Among patients with SSc, cancer risk is particularly high in men, and, for lung cancer, in individuals with interstitial lung disease, but there are no clear data suggesting an association of higher neoplastic risk and SSc-specific autoantibody status.

In a retrospective evaluation of 360 Italian patients with $\mathrm{SSc}$ and isolated positivity for one of the 3 main SSc-specific autoantibodies, we observed a history of malignancies in $10.8 \%$ of the subjects. These raw data are similar to data observed by others ${ }^{8,18,19}$, but direct comparisons are not possible because of different patient selection and population characteristics. In particular, our series includes a higher percentage of women and ACA-positive individuals compared with patients from Northern Europe or the US.

More importantly, the number of malignancy cases diagnosed in our patients with anti-RNAP III antibodies was significantly higher than in those with anti-topo I antibodies or ACA ( $p<0.001)$. The percentage of patients having a malignancy diagnosed before onset of SSc, or more than 1 year after that date, was not significantly different among the 3 groups. The association of malignancy with anti-RNAP III antibodies seems to be explained mainly by the higher frequency of patients in which cancer was synchronous to SSc (i.e., diagnosed 6 months before to less than 12 months after the onset of SSc; $p<0.001$ compared to other autoantibody groups).
Our observation therefore confirms, in a different population and with a different approach, the findings of Shah, et $a l^{8}$, who first demonstrated a close temporal relationship between onset of SSc and diagnosis of malignancy in American anti-RNAP III-positive SSc patients with cancer. The clinical characteristics of the 3 anti-RNAP III-positive patients with synchronous diagnosis of cancer and SSc presented here are also very similar to those reported by Shah, et $a l^{8}$, with rapid progression of diffuse skin involvement and a high frequency of severe organ involvement.

The association of positivity for anti-RNAP III with malignancies synchronous to onset of SSc might have clinically and biologically relevant implications.

From a clinical point of view, our data suggest that the risk of cancer diagnosis at the time of SSc onset in patients with anti-RNAP III antibodies may be relatively high [3/16 (18.8\%); 95\% CI 5.8-43.8], perhaps similar to what has been observed in patients with dermatomyositis ${ }^{20}$, and that patients with rapid progression of skin involvement seem to be particularly at risk, as suggested ${ }^{8}$. We feel that at least in patients with these clinical characteristics (no interval between the appearance of Raynaud's phenomenon and skin involvement, aggressive cutaneous disease), investigation for anti-RNAP III antibodies is mandatory and an active search for malignancy is warranted.

As well, this association suggests possible pathogenic pathways to be addressed in future studies. Shah, et al demonstrated enhanced RNAP III antigen nucleolar staining in tumor tissues from SSc patients with anti-RNAP III antibodies, and hypothesized that tumors expressing high concentrations of RNAP III may initiate an immune response to these antigens ${ }^{8}$. The enhanced expression of the same antigens (e.g., in damaged blood vessels) might be the link between anti-tumoral and autoimmune response, which being directed against host tissues may thereafter also lead to the appearance of clinical features of $\mathrm{SSc}^{8}$. It should be considered that not only RNAP III but also other autoantigens targeted by SSc autoantibodies can be expressed by cancer cells, and, under certain circumstances, also by normal cell surfaces ${ }^{21}$. Moreover, Kuwana, et $a l^{22}$ reported 2 patients with long-standing SSc in whom the anti-topo I antibody response was greatly enhanced at the time of diagnosis of lung cancer, and this observation supports the possibility that tumor antigens may drive autoantibody production. The typical clinical characteristics of many anti-RNAP III-positive patients with $\mathrm{SSc}^{1,5,6,7}$ seem to support a model in which an event induces a tumultuous, rapidly evolving pathogenic process. The association, in some cases, with a simultaneously growing cancer suggests the hypothesis that a tumor antigen-driven autoimmune response might be one of the factors involved in this process.

Our study has several limitations. Not all the anti-topo I or ACA-positive patients were tested (or retested at the time of cancer diagnosis) for anti-RNAP III antibodies. However,

Personal non-commercial use only. The Journal of Rheumatology Copyright (C) 2011. All rights reserved. 
in our experience, and as reported by previous investigators, SSc-specific antibodies are often mutually exclusive, and change from one to another during the disease course is exceptional (as reviewed ${ }^{1,16}$ ). More important limitations are the retrospective design of the study and the relatively small number of patients. However, the median followup of the entire study cohort was long, 118 months, and the number of patients lost to followup or moved to other centers was relatively small (Table 1). This should reduce selection biases and, indeed, the raw frequency of malignancies detected in our populations of SSc patients was similar to those observed in other studies in patients at higher risk ${ }^{8,18}$. In our opinion, the main result of our study, the association of positivity for anti-RNAP III antibodies with malignancies synchronous to onset of SSc in Italian patients, can therefore be considered reliable, and reinforces findings from a cohort of American patients ${ }^{8}$. This suggests that further prospective, possibly multicenter studies analyzing larger numbers of patients to better define this important issue are warranted.

\section{REFERENCES}

1. Steen VD. The many faces of scleroderma. Rheum Dis Clin North Am 2008;34:1-15.

2. Okano Y, Steen VD, Medsger TA Jr. Autoantibody reactive with RNA polymerase III in systemic sclerosis. Ann Intern Med 1993;119:1005-13.

3. Bunn CC, Denton CP, Shi-Wen X, Knight C, Black CM. Anti-RNA polymerases and other autoantibody specificities in systemic sclerosis. Br J Rheumatol 1998;37:15-20.

4. Ingraham KM, O'Brien MS, Shenin M, Derk CT, Steen V. Gastric antral vascular ectasia in systemic sclerosis: demographics and disease predictors. J Rheumatol 2010;37:603-7.

5. Ceribelli A, Cavazzana I, Airo P, Franceschini F. Anti-RNA polymerase III antibodies as a risk marker for early gastric antral vascular ectasia (GAVE) in systemic sclerosis. J Rheumatol 2010;37:1544.

6. Cavazzana I, Ceribelli A, Airo P, Zingarelli S, Tincani A, Franceschini F. Anti-RNA polymerase III antibodies: a marker of systemic sclerosis with rapid onset and skin thickening progression. Autoimmun Rev 2009;8:580-4.

7. Codullo V, Cavazzana I, Bonino C, Alpini C, Cavagna L, Cozzi F, et al. Serologic profile and mortality rates of scleroderma renal crisis in Italy. J Rheumatol 2009;36:1464-9.

8. Shah AA, Rosen A, Hummers L, Wigley F, Casciola-Rosen L. Close temporal relationship between onset of cancer and scleroderma in patients with RNA polymerase I/III antibodies. Arthritis Rheum 2010;62:2787-95.
9. Meyer O, Fertig N, Lucas M, Somogyi N, Medsger TA Jr. Disease subsets, antinuclear antibody profile, and clinical features in 127 French and 247 US adult patients with systemic sclerosis. J Rheumatol 2007;34:104-9.

10. Meyer O, De Chaisemartin L, Nicaise-Roland P, Cabane J, Tubach F, Dieude P, et al. Anti-RNA polymerase III antibody prevalence and associated clinical manifestations in a large series of French patients with systemic sclerosis: a cross-sectional study. J Rheumatol 2010;37:125-30.

11. Codullo V, Morozzi G, Bardoni A, Salvini R, Deleonardi G, De Pita $\mathrm{O}$, et al. Validation of a new immunoenzymatic method to detect antibodies to RNA polymerase III in systemic sclerosis. Clin Exp Rheumatol 2007;25:373-7.

12. LeRoy EC, Medsger TA Jr. Criteria for the classification of early systemic sclerosis. J Rheumatol 2001;28:1573-6.

13. Subcommittee for Scleroderma Criteria of the American Rheumatism Association Diagnostic Therapeutic Criteria Committee. Preliminary criteria for the classification of systemic sclerosis (scleroderma). Arthritis Rheum 1980;23:581-90.

14. Valentini G, Medsger TA Jr, Silman AJ, Bombardieri S. The assessment of the patient with systemic sclerosis. Clin Exp Rheumatol 2003;Suppl 29:S1-S56.

15. Medsger TA Jr, Silman AJ, Steen VD, Black CM, Akesson A, Bacon PA, et al. A disease severity scale for systemic sclerosis: development and testing. J Rheumatol 1999;26:2159-67.

16. Ho KT, Reveille JD. The clinical relevance of autoantibodies in scleroderma. Arthtritis Res Ther 2003;5:80-93.

17. Bosello S, De Santis M, Lama G, Spano C, Angelucci C, Tolusso B, et al. B cell depletion in diffuse progressive systemic sclerosis: safety, skin score modification and IL-6 modulation in an up to thirty-six months follow-up open-label trial. Arthritis Res Ther 2010;12:R54.

18. Rosenthal AK, McLaughlin JK, Gridley G, Nyrén O. Incidence of cancer among patients with systemic sclerosis. Cancer 1995;76:910-4.

19. Olesen AB, Svaerke C, Farkas DK, Sørensen HT. Systemic sclerosis and the risk of cancer: a nationwide population-based cohort study. Br J Dermatol 2010;163:800-6.

20. Hill CL, Zhang Y, Sigurgeirsson B, Pukkala E, Mellemkjaer L, Airio A, et al. Frequency of specific cancer types in dermatomyositis and polymyositis: a population-based study. Lancet 2001;357:96-100.

21. Henault J, Robitaille G, Senecal JL, Raymond Y. DNA topoisomerase I binding to fibroblasts induces monocyte adhesion and activation in the presence of anti-topoisomerase I autoantibodies from systemic sclerosis patients. Arthritis Rheum 2006;54:963-73.

22. Kuwana M, Fujii T, Mimori T, Kaburaki J. Enhancement of anti-DNA topoisomerase I autoantibody response after lung cancer in patients with systemic sclerosis. A report of two cases. Arthritis Rheum 1996;39:686-91. 